\title{
Influence of post-thaw culture on the cleavage stage embryo development and the pregnancy rate
}

\author{
Escalante Elisa; Moreno Pilar; Ruiz Nerea; Abad Magdalena; de la Fuente Laura. \\ Human Reproduction Unit Obstetrics \& Gynaecology. Hospital 12 de Octubre. Madrid. Spain.
}

Study question: Does the overnight culture improve the results of cryotransfer with slow freezing protocol in cleavage stage embryos?

Summary answer: No advantage could be found for a 20-24 hours post-thaw culture period, even for embryos with observed mitotic activity.

What is known already: Cryotransfers success rate is related with many known factors like embryo quality, maternal age, number of transferred embryos and implantation potential determinate by blastomere survival and embryo development. Many authors have related mitotic resumption with better prognosis and stop embryo development with lower success. Overnight culture is an extended procedure for the frozen embryo transfer (FET) but the in vitro culture conditions may not exactly replace the physiological environment. In the other hand, short embryo culture (2-4 hours), which is also common, could be associated with a higher percentage of no mitotic resumption embryos transferred.

Study design, size, duration: During 2014-2016 period, 244 freezing-thawing cycles were performed at the Human Reproduction Unit of Hospital Universitario 12 de Octubre, Madrid, Spain, in a public IVF programme without egg-donation. Three study groups with different freezing day and culture conditions were compared regarding pregnancy rate. 130 cycles had freezing, thawing and transfer on day 3 (group 1), 56 on day 2 (group 2) and 59 had freezing and thawing on day 2 and transfer on day 3.

Participants/materials, setting, methods: 182 Patients who underwent cryotransfer cycles after IVF were included and only patients with uterine anomalies were excluded. All the studied embryos were freeze and thaw with the slow protocol and transferred only when $\geq 50 \%$ of the initial number of blastomeres remained intact. Transfers were performed when the endometrium reached $8 \mathrm{~mm}$ after hormonal replacement treatment with oestragens/progesterone. Clinical pregnancy was defined by the presence of a gestational sac on ultrasound examination.

Main results and the role of chance: Daily laboratory programme rules the embryo thawing protocol election according to assistance requirements in order to ovoid overcrowded activity. We select between a few hours culture (2-4h) and an overnight culture (20-24h) because nowadays we still haven't found a standardized criteria about the effectiveness of one strategy over another. The maternal age at ovarian pick-up, embryo quality and post-thaw embryo survival rate were not statistically different between groups $(p>0,01)$. Although no differences could be detected in those determining factors, outcomes are clearly influenced by the culture period. Pregnancy rates per transfer were statistically different between groups ( $p=0,005): 25,23 \%$ (group 1$)$, 33, $33 \%$ (group 2) and 7, 55\% (group 3). This retrospective analysis indicated that short post-thaw culture period is associated with higher pregnancy rate. As consequence, our results support the survival blastomere rate as the most determining factor to select embryos for transfer while longer post-thaw cultures are an extra charge for the laboratory and don't seem to be particularly benefit in terms of pregnancy rates.

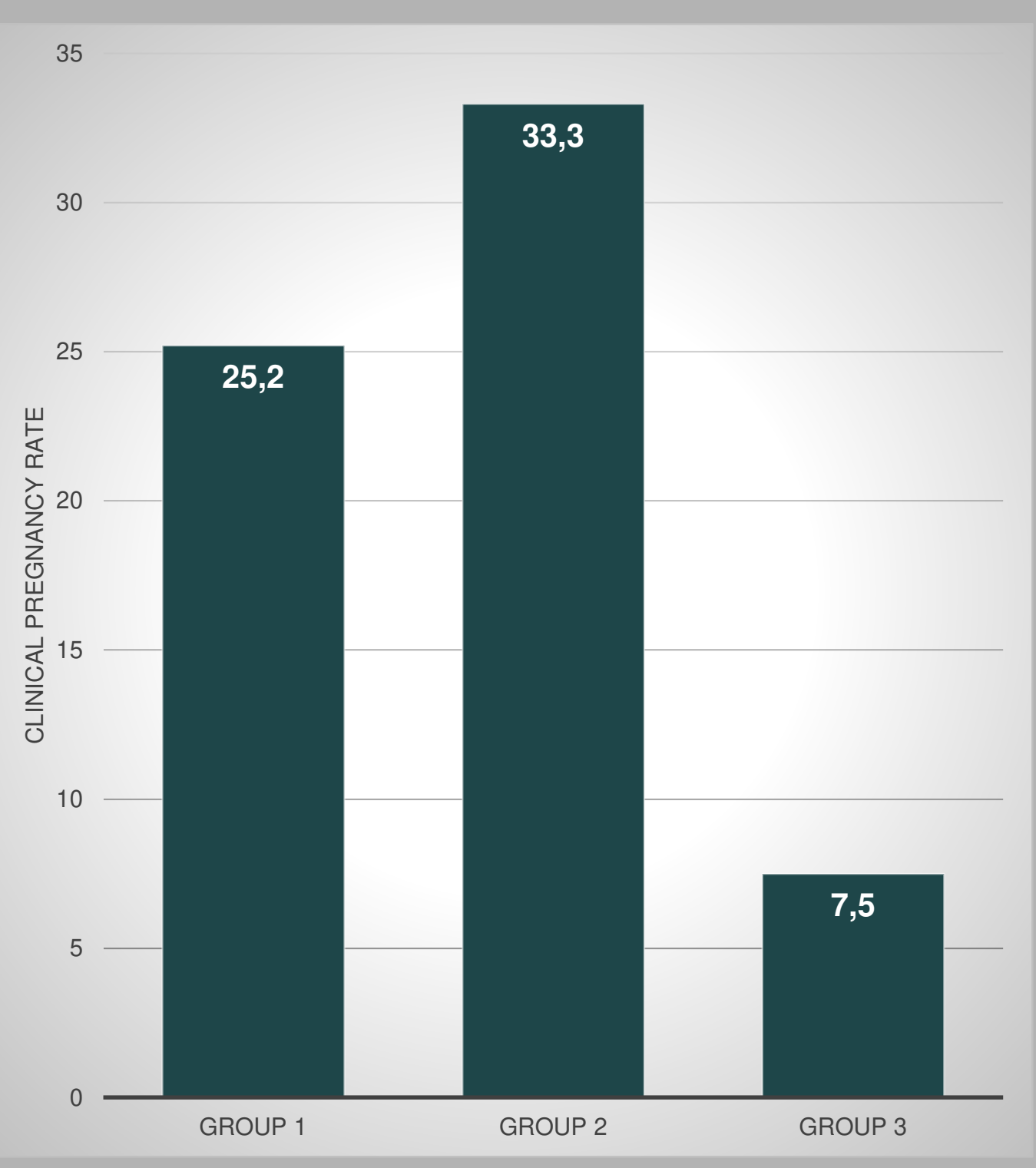

Conclusions: As no advantage could observed after overnight day 2 to day 3 post-thawing embryo culture, short culture (2-4 hours) appears to be the best option in terms of pregnancy rates. 